\title{
Anorectal Malformation with Rectovestibular Fistula and Vaginal Agenesis: Usage of Rectovestibular Fistula as a Neovagina Followed by PSARP with Preservation of the Anal Sphincter
}

\author{
Antonio Dessanti ${ }^{1}$ Osnel Louisma ${ }^{2}$ Gabriel Steve Pierre ${ }^{2} \quad$ Nguhien Thanh Liem $^{3}$ \\ ${ }^{1}$ AOU University Hospital, University of Sassari, Sassari, Italy \\ 2 Department of Pediatric Surgery, Saint Damien Children Hospital, \\ Port Au Prince, Haiti \\ Address for correspondence Antonio Dessanti, Pediatric Surgery, \\ AOU University Hospital, University of Sassari, Via Verona 24, Sassari \\ 07100, Italy (e-mail: antoniodessanti@yahoo.it).
}

${ }^{3}$ Department of Pediatric Surgery, National Children Hospital and Vinmec International Hospital, Hanoi, Vietnam

European J Pediatr Surg Rep 2021;9:e51-e55.

\author{
Abstract \\ Keywords \\ - anorectal \\ malformation \\ - vaginal agenesis \\ - posterior sagittal \\ anorectoplasty \\ - vaginoplasty
}

Anorectal malformation with rectovestibular fistula associated with vaginal agenesis is rare. We report on a child in whom this combination was diagnosed at the age of 1 year. After creation of a divided descending colostomy, we chose to leave the rectumrectovestibular fistula to function as a neovagina, while the sigmoid colon was relocated via modified posterior sagittal anorectoplasty. The colostomy was closed 6 months after the main surgery. After a follow-up of 3 years, the patient is continent for stool and urine. The introitus of the neovagina appears wide and easy to explore. We conclude that our surgical approach may be a good option for these children.

\section{New Insights and the Importance for the Pediatric Surgeon}

ARM is one of the most complex congenital anomalies involving several organs. The association of ARM with vaginal genesis, as it is described in our case, is a very rare and complex event to be corrected surgically.

\section{Introduction}

Anorectal malformations (ARMs) are rare congenital defects with an estimated worldwide incidence of 2 to 6 per 10,000 live births. ${ }^{1}$ They comprise a wide range of pathologies that may involve other parts of the gastrointestinal tract as well as urinary and genital tracts. ${ }^{2-9}$ The most common type of ARM in female patients is a rectovestibular fistula. The incidence of vaginal agenesis associated with ARM is even lower. ${ }^{4}$ The approach to this combination was first described by Cohn and Murphy via a combined laparotomic and perineal operation. ${ }^{10}$ The use of rectovestibular fistula as a neovagina has also been reported in combination of a

received

June 18,2020

accepted after revision

March 6, 2021
DOI https://doi.org/

$10.1055 / \mathrm{s}-0041-1728725$.

ISSN 2194-7619. posterior sagittal anorectoplasty (PSARP), ${ }^{2}$ as described by Levitt et al. ${ }^{4}$ We describe the case of a 1-year-old girl with ARM and rectovestibular fistula in whom we chose to leave the rectum-rectovestibular fistula to function as a neovagina, while the sigmoid colon was relocated via modified PSARP, according to Liem and Hau, and Liem and Quynh technique. ${ }^{11,12}$

\section{Case Report}

The previous term infant (birth weight $3.1 \mathrm{~kg}$ ) was admitted to the Pediatric Surgery Service at Saint Damien Pediatric Hospital, Port-au-Prince, Haiti, with a diagnosis of vaginal

(c) 2021. The Author(s).

This is an open access article published by Thieme under the terms of the Creative Commons Attribution License, permitting unrestricted use, distribution, and reproduction so long as the original work is properly cited. (https://creativecommons.org/licenses/by/4.0/)

Georg Thieme Verlag KG, Rüdigerstraße 14, 70469 Stuttgart, Germany 
agenesis associated to ARM with rectovestibular fistula. Until 10 months of age, the infant defecated through the rectovestibular fistula that required dilations even though it had a considerable caliber. Two months prior to clinical admission, she received a descending colostomy with distal mucous fistula. No other obvious malformations and a normal sacrum were found. Due to the lack of resources at the local hospital, no additional diagnostic imaging, for example, magnetic resonance imaging, was performed.

We chose to leave the rectovestibular fistula in situ to function as a neovagina and to perform a PSARP, ${ }^{2,4}$ modified at preserving the anal sphincter as reported by Liem and Hau, and Liem and Quynh. ${ }^{11,12}$ The patient was placed in a prone jackknife position. After locating the anal sphincter by using a neurostimulator, a posterior sagittal incision of the cutaneous and subcutaneous planes was made 1 to $2 \mathrm{~cm}$ over the coccyx to the superior limit area of the anal dimple. The dissection was deepened while paying attention to the midline, to avoid injuries to muscles and nerve fibers, and arrested at the level of the upper limit of the muscle complex. The muscle complex was identified and its lower part was retracted downward to expose the posterior wall of the rectum ( - Fig. 1). The fibrotic bands between the rectal wall and the coccyx were divided and the coccyx removed aiming at gaining extra space. The rectum appeared considerably dilated to the extent that not all its circumferences could be isolated. As a consequence, it was isolated only from its lateral sides and the anterior surface of the sacrum until the peritoneal reflection which was opened in view of the subsequent laparotomy.

After having temporarily closed the posterior sagittal skin incision to minimize the risk of bacterial contamination, the patient was placed in supine position to open the abdomen through a Pfannenstiel incision. The rectum, which was abnormally dilated until 1 to $2 \mathrm{~cm}$ beyond the peritoneal reflection, continued as sigmoid colon whose caliber appeared suddenly normal ( - Fig. 2). The rectouterine Douglas' pouch was explored; while ovaries were located with fallopian tubes presenting cordlike structures, instead no structures resembling vagina and uterus were found.

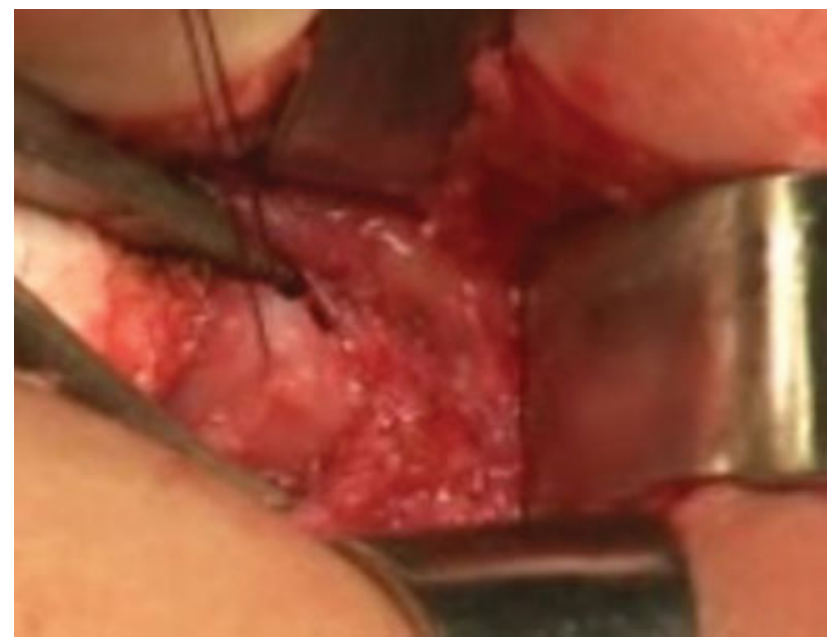

Fig. 1 The lower part of the muscle complex was retracted down to expose the posterior wall of the rectum.

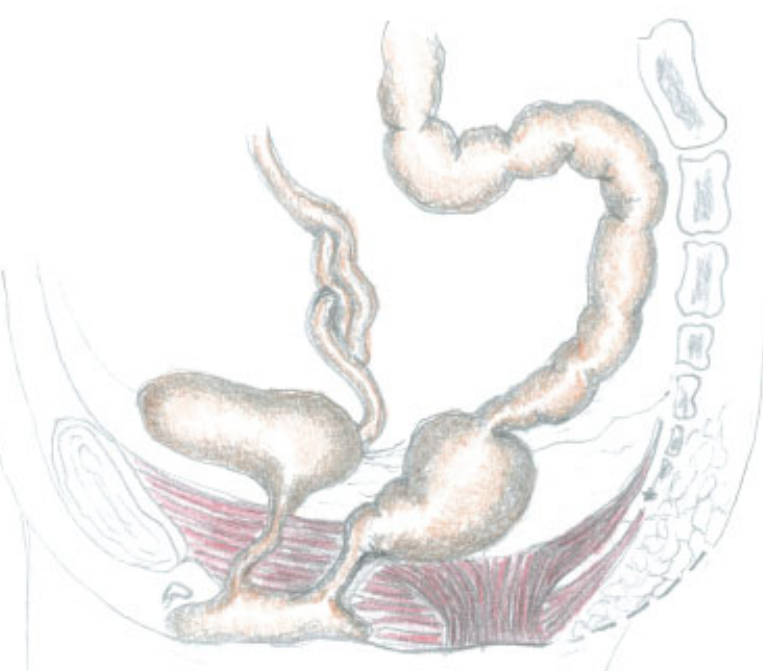

Fig. 2 Anatomy of the patient.

The dilated rectum was surgically separated from the sigmoid colon and closed; the sigmoid colon was only temporarily sutured to avoid fecal contamination (-Fig. 3 ).

After binding and dissecting some branches of its vascular arcade, the sigmoid colon was then mobilized, as it needed to be easily passed without traction through the retrorectal space into the previously opened posterior sagittal space (-Fig. 4), which was reopened at this point.

Once the suprapubic incision was sutured and the patient was again in the same prone position as before, a second small inverted cutaneous Y-shape incision was made on the anal dimple, after reconfirming the location of the underlying anal muscle complex using the neurostimulator (-Fig.5). Our technique was different from the original technique of PSARP described by Peña and Devries, which envisages to separate the sphincter muscle complex in the midline. ${ }^{2}$ In contrast, we created a tunnel in the center of the sphincter muscle complex through a gentle and progressive dilation of its muscle fibers using Hegar dilators (size 6-12) (-Fig. 6)

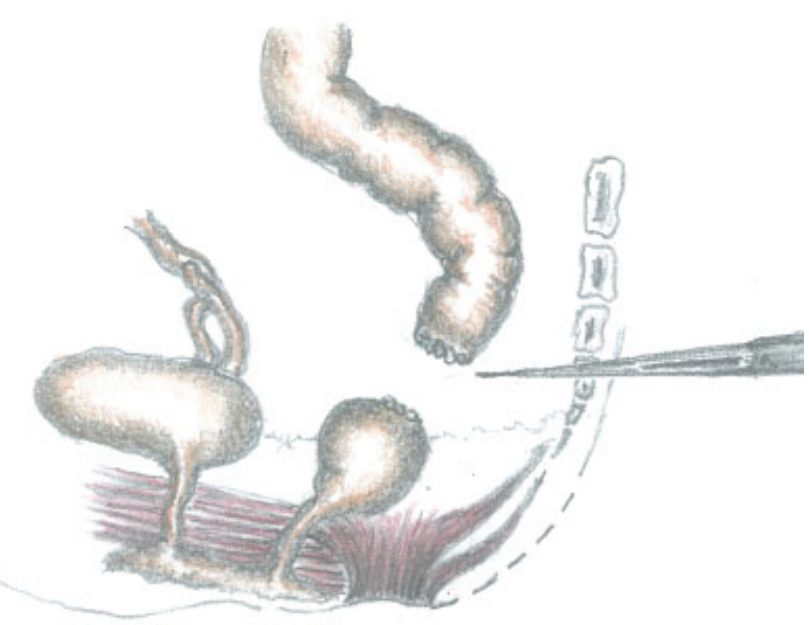

Fig. 3 Sigmoid colon surgically separated from the dilated rectum. 


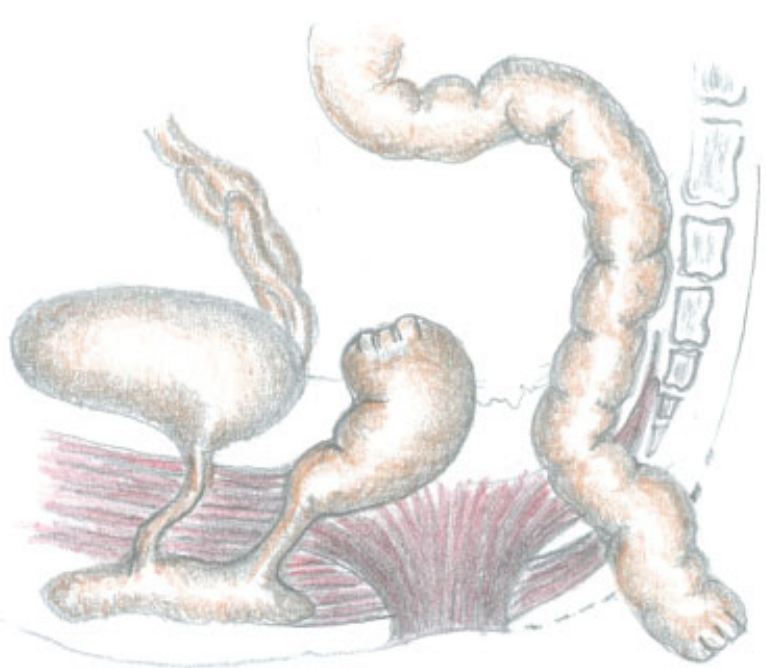

Fig. 4 Mobilized sigmoid colon passed into the posterior sagittal space.

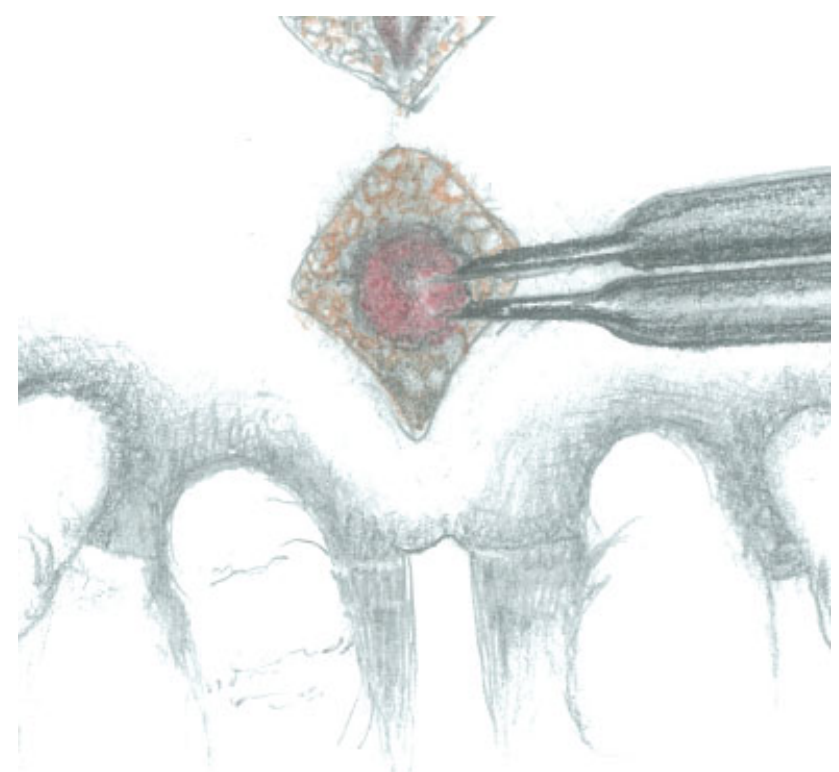

Fig. 5 The center of the sphincter complex is defined using a neurostimulator.

until reaching an acceptable diameter to enable the mobilized sigmoid colon to go through it.

Consequently, the sigmoid colon, that managed to pass through the dilated tunnel of the sphincter muscle complex, was sutured to the external sphincter and to the margins of the small Y-shape cutaneous incision that had already been made in the anal dimple by using interrupted absorbable stitches (-Fig. 7).The posterior sagittal incision was then closed. Postoperatively, the neoanus was gradually dilated using Hegar dilators from the second week after the surgery for a total period of 1 month. The colostomy was closed after 6 months.

No complications were observed during the early postoperative period. Neither anal stenosis nor other local and general complications were observed.

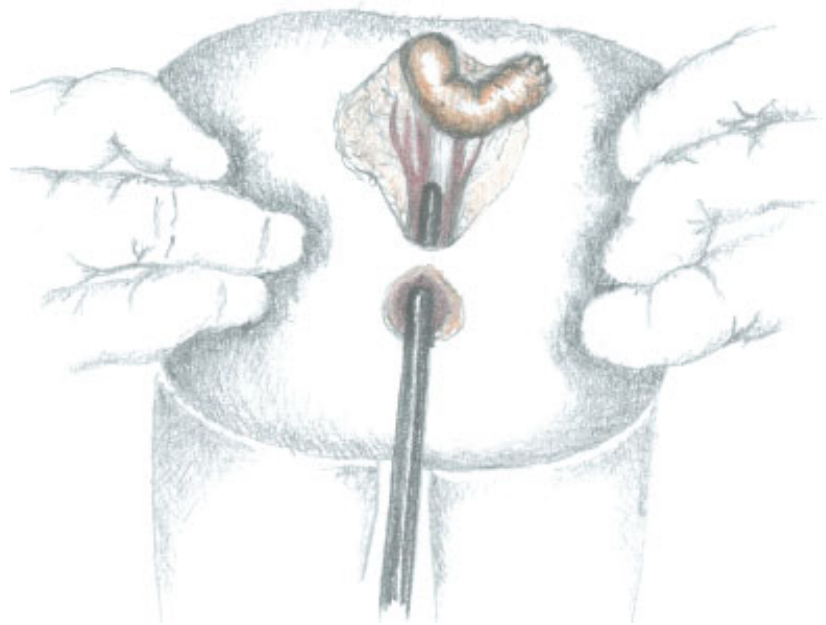

Fig. 6 Progressive dilation of the tunnel created in the center of the sphincter complex.

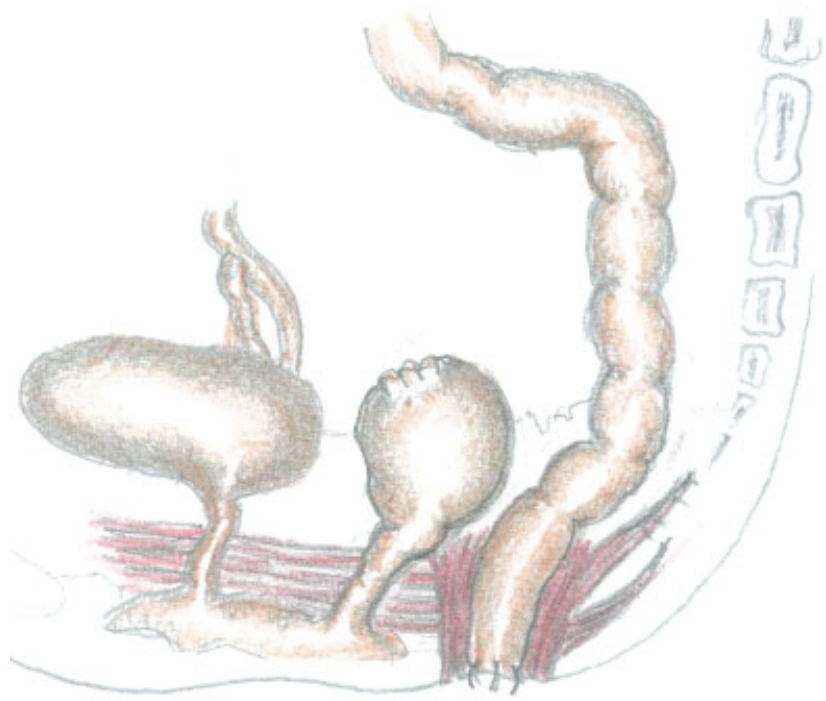

Fig. 7 The sigmoid colon is passed through the dilated tunnel of the sphincter complex and a neoanus is created.

Two years after the anoplasty and at the age of 3 years, the parents reported that a few months earlier the girl stopped using diapers as she had achieved good bowel control and always evacuated spontaneously. So, no complications occurred such as fecal incontinence (not even partially), no constipation or mucosal prolapse, nor urinary incontinence. The rectovestibular fistula, that had become the vagina introitus, appeared relatively wide and easily to explore (-Fig. 8).

\section{Discussion}

Our case is a subtype of Mayer-Rokitansky-Kuster-Hauser syndrome, ${ }^{6,13}$ caused by embryologic underdevelopment of the Mullerian duct with resultant agenesis of vagina and uterus. Vaginal agenesis associated with ARM is uncommonly seen. ${ }^{4-7,9,10,13-17}$ Banu et a ${ }^{18}$ reported 5 patients with a 


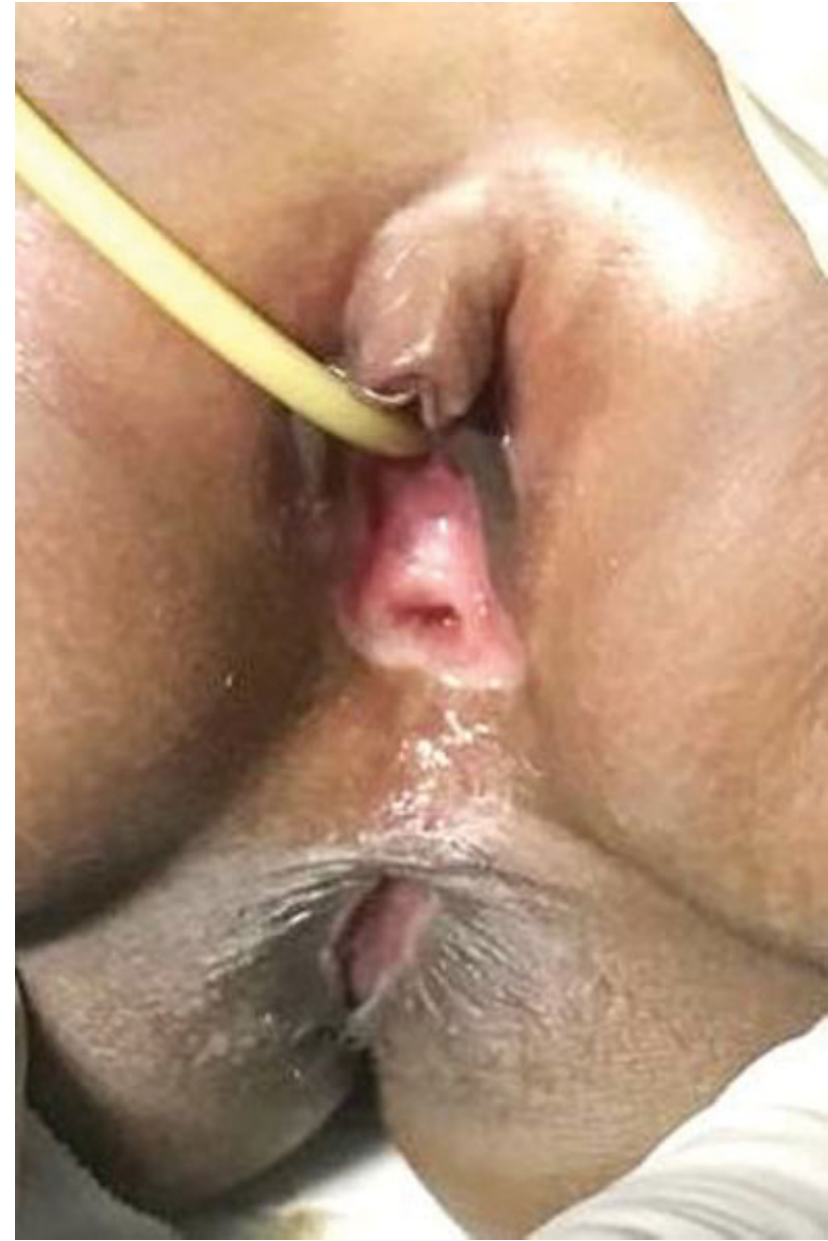

Fig. 8 Neoanus and neovaginal introitus at 2.6 years of age.

rectovestibular fistula and absence of vagina in their series of 563 cases.

For what concerns the reconstruction of vaginal agenesis in cases of vaginal agenesis without ARM, the first-line approach, based on safety reasons, is the primary vaginal elongation by dilations, sometimes with local estrogen, known as Vecchietti procedure ${ }^{13,19}$; if no satisfactory results are achieved, during adolescence can be indicated an intraabdominal traction applied to the perineal membrane, causing invagination over the course of a week. This technique often produces an inadequate vaginal length that might interfere with normal sexual life. Due to these reasons, other surgical options include vaginoplasty using a part of the ileus or sigmoid colon. ${ }^{5-7,9,13,15,16}$

In 1956, Cohn and Murphy ${ }^{10}$ proposed for the time to correct vaginal agenesis in ARM rectovestibular fistula type, using the rectum-rectovestibular fistula in situ as a neovagina. In other reports on vaginal agenesis, an abdominoperineal approach ${ }^{14,17}$ and a PSARP according to Levitt et al were used. ${ }^{4,20}$

This was also our decision: the vaginal agenesis was corrected using the rectum including the rectovestibular fistula to function as the vagina. The sigmoid colon was pulled through and relocated to be the neoanus, ${ }^{2}$ as proposed by Levitt et al, ${ }^{4}$ including laparotomy, but preserving the anal sphincter, according to Liem and Hau, and Liem and Quynh. ${ }^{11,12}$

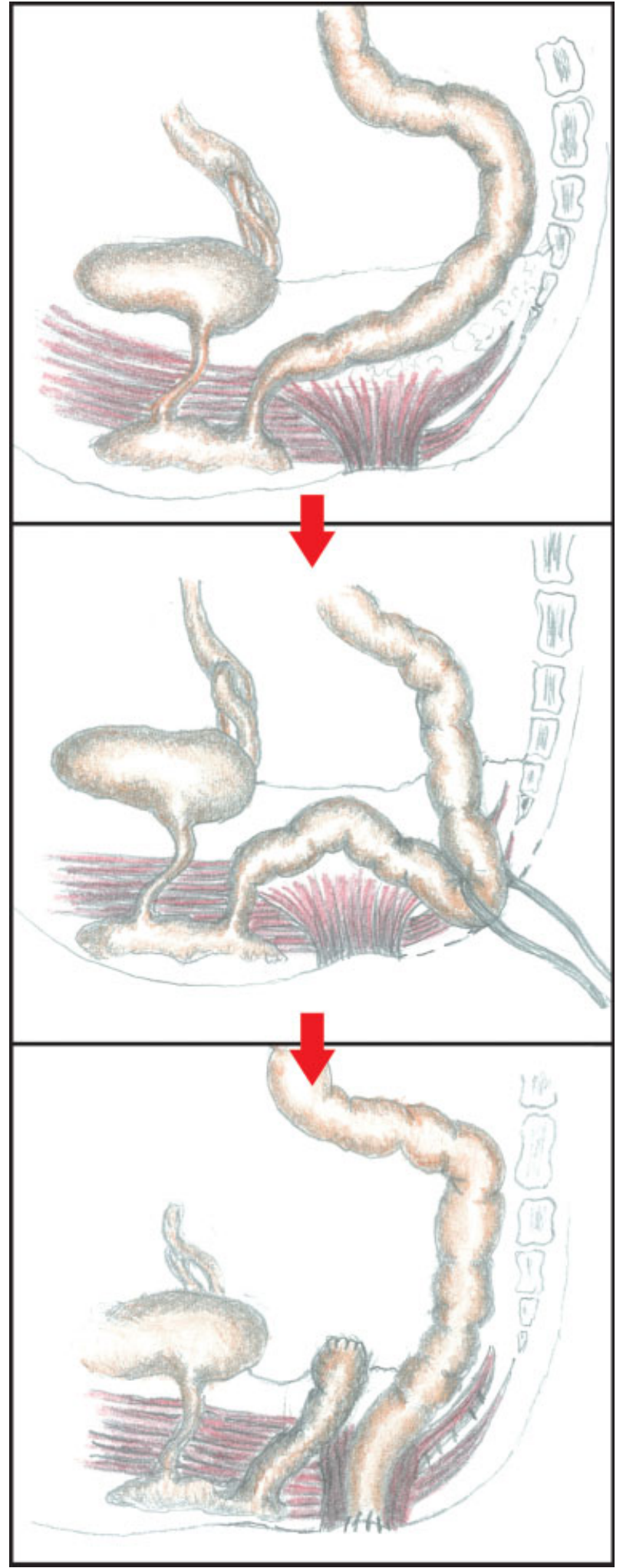

Fig. 9 Sketch of the one-stage posterosagittal approach, preserving the muscular sphincter complex (Liem and Hau, and Liem and Quynh technique), to correct vaginal agenesis and anorectal malformation with rectovestibular fistula.

At 3 years of age follow-up, the patient showed good bowel control, which generally at that age cannot be completely achieved. ${ }^{21}$ No clinical complications such as constipation or fecal incontinence were reported. 
Although it represents a unique case, we believe that such satisfactory functional results probably also derived from the surgical technique used besides the fact that no lesions involving the sacrum were observed; unlike what takes place in the Peña and Devries' PSARP technique, based on an incision of the sphincter muscle complex in the midline, ${ }^{2}$ the sphincter muscle complex was not divided and the sigmoid colon was pulled through a tunnel created at its center. Having left the sphincter muscle complex untouched leads us to hypothesize that the function of this important structure was preserved; this technique has already been reported. ${ }^{11,12}$

Differently from our strategy of using the in situ rectum as a neovagina, another clinical option was to separate the rectum from urethra and relocate it within the limits of the sphincter mechanism. However, the major disadvantage of this technique is that later a more time-consuming surgery for vaginal reconstruction is required, which can also be an intervention of higher complexity. In addition, the separation of the rectum from the urethra might provoke denervation of the lower urinary tract as well as risks of denervation involving the rectum. ${ }^{4}$

As the rectum shoved a considerable dilation, we added a suprapubic laparotomy before performing a modified PSARP with preservation of an intact anal sphincter. If the rectum had a normal caliber, we would have pulled down the proximal rectum to the neoanus (-Fig.9).

The final result of our procedure was an introitus situated rather anteriorly in the vulva probably due to extra space left by the missing vagina whose space might have been taken.

We hope that the future sexual function and satisfaction will be beneficial as the terminal nerves to the vulva and rectum involved were not subjected to any surgical manipulation, unlike a lot of other surgical techniques currently used for vaginal reconstruction.

Clearly, the diameter of the new vaginal introitus will have to be assessed during puberty; in the case of insufficient diameter, an event highly expected to occur, it might be widened using the wide walls of the rectovestibular fistula.

Also, at long-term follow-up, the sensitivity of the new vaginal introitus will have to be assessed.

\section{Conclusion}

Our surgical strategy based on the decision to leave the rectum in situ with its rectovestibular fistula used as a neovagina before creating a neoanus via a PSARP may provide an effective alternative to other vaginal reconstruction strategies using more complex reconstruction techniques.

\section{Conflict of Interest}

None declared.

\section{Acknowledgments}

We wish to thank Prof. Salvatore Dessole, Director of Obstetrics and Gynecology Clinic, Sassari University, for valuable suggestion given. Maria Teresa Ara for her support in providing the anatomical sketches and Giustina Casu Finlayson who checked English language and editing.

\section{References}

1 de Blaauw I, Wijers CHW, Schmiedeke E, et al. First results of a European multi-center registry of patients with anorectal malformations. J Pediatr Surg 2013;48(12):2530-2535

2 Peña A, Devries PA. Posterior sagittal anorectoplasty: important technical considerations and new applications. J Pediatr Surg 2020;30(05):447-451

3 Banu T, Karim A, Adel MG, et al. Multicenter study of 342 anorectal malformation patients: age, gender, Krickenbeck subtypes, and associated anomalies. Eur J Pediatr Surg 2020; 30:447-451

4 Levitt MA, Stein DM, Peña A. Rectovestibular fistula with absent vagina: a unique anorectal malformation. J Pediatr Surg 1998;33 (07):986-989, discussion 990

5 Wesley JR, Coran AG. Intestinal vaginoplasty for congenital absence of the vagina. J Pediatr Surg 1992;27(07):885-889

6 Wester T, Tovar JA, Rintala RJ. Vaginal agenesis or distal vaginal atresia associated with anorectal malformations. J Pediatr Surg 2012;47:571-576

7 Pandya KA, Koga H, Okawada M, Coran AG, Yamataka A, Teitelbaum DH. Vaginal anomalies and atresia associated with imperforate anus: diagnosis and surgical management. J Pediatr Surg 2015;50(03):431-437

8 Vilanova-Sanchez A, Reck CA, McCracken KA, et al. Gynecologic anatomic abnormalities following anorectal malformations repair. J Pediatr Surg 2018;53(04):698-703

9 Skerritt C, Vilanova Sánchez A, Lane VA, et al. Menstrual, sexual, and obstetrical outcomes after vaginal replacement for vaginal atresia associated with anorectal malformation. Eur J Pediatr Surg 2017;27(06):495-502

10 Cohn BD, Murphy DR. Imperforate anus with agenesis of the vagina. Ann Surg 1956;143(03):430-432

11 Liem NT, Hau BD. Long-term follow-up results of the treatment of high and intermediate anorectal malformations using a modified technique of posterior sagittal anorectoplasty. Eur J Pediatr Surg 2001;11(04):242-245

12 Liem NT, Quynh TA. One stage operation through modified posterior sagittal approach preserving the sphincter intact for anal agenesis with rectovestibular fistula. J Pediatr Surg 2015;50 (04):634-637

13 Committee on Adolescent Health Care. Mullerian agenesis: diagnosis, management and treatment. Obstet Gynecol 2018;131(01): e35-e42

14 Ein SH, Stephens CA. Vaginal construction in children with absent vagina and imperforate anus. J Pediatr Surg 1971;6(04):435-439

15 De la Torre L, Cogley K, Calisto JL, Santos K, Ruiz A, Zornoza M. Vaginal agenesis and rectovestibular fistula. Experience utilizing distal ileum for the vaginal replacement in these patients, preserving the natural fecal reservoir. J Pediatr Surg 2016;51(11):1871-1876

16 Günşar C, Genç A, Sencan A, Dağlar Z, Alparslan O, Mir E. MURCS association and rectovestibular fistula: case report of a patient treated with one-stage posterior sagittal anorectoplasty and sigmoid loop vaginoplasty. J Pediatr Surg 2003;38(02):262-264

17 Fujiwara Y, Oizumi T, Sasahara M, Kato E, Kakizaki G. A case of congenital imperforate anus and absent vagina with a functioning uterus. Tohoku J Exp Med 1974;113(03):283-289

18 Banu T, Hannan MJ, Aziz MA, Hoque M, Laila K. Rectovestibular fistula with vaginal malformations. Pediatr Surg Int 2006;22(03):263-266

19 Vecchietti G. Neovagina nella sindrome di Rokitansky-KüsterHauser. Attual Ostet Ginecol 1965;11(02):131-147

20 Levitt MA, Bischoff A, Breech L, Peña A. Rectovestibular fistulararely recognized associated gynecologic anomalies. J Pediatr Surg 2009;44(06):1261-1267, discussion 1267

21 Issenman RM, Filmer RB, Gorski PA. A review of bowel and bladder control development in children: how gastrointestinal and urologic conditions relate to problems in toilet training. Pediatrics 1999;103(6 Pt 2):1346-1352 